\title{
Enquête
}

Archives de la revue Enquête

5 | 1989

Biographie et cycle de vie

\section{L'hypothétique consensus familial sur la socialisation des parcours de vie}

\section{Annette Langevin}

\section{(2) OpenEdition}

Journals

Édition électronique

URL : http://journals.openedition.org/enquete/92

DOI : 10.4000/enquete.92

ISSN : 1953-809X

Éditeur :

Cercom, Éditions Parenthèses

Édition imprimée

Date de publication : 2 mars 1989

\section{Référence électronique}

Annette Langevin, "L'hypothétique consensus familial sur la socialisation des parcours de vie »,

Enquête [En ligne], 5 | 1989, mis en ligne le 27 juin 2013, consulté le 19 avril 2019. URL : http:// journals.openedition.org/enquete/92 ; DOI : 10.4000/enquete.92

Ce document a été généré automatiquement le 19 avril 2019 


\title{
L'hypothétique consensus familial sur la socialisation des parcours de vie
}

\author{
Annette Langevin
}

1 Remettre en cause la notion de cycle c'est faire référence à trois niveaux d'appréhension : le cycle dans son sens le plus trivial en tant que système de représentation topologique, qui non seulement situe dans un espace mais organise qualitativement cette représentation spatio-temporelle; en second, l'emploi du cycle en tant que méthode de classification d'une dynamique; et, enfin, le concept de "cycle» dans les sciences humaines. La méthode et le concept de «cycle de la vie familiale» ont fait l'objet de nombreux débats. C'est dans la mouvance de ces controverses que s'inscrit cette réflexion sur le rôle du microcosme familial dans la socialisation des durées et des rythmes des parcours de vie tels qu'ils sont captés dans le recueil de récits autobiographiques.

\section{Le cycle : système de représentation spatio-temporel}

2 Le cycle tel qu'en lui-même propose un déroulement perpétuellement reconduit. L'idée de déroulement cyclique s'oppose à celle d'historicité, la chronologie des faits se reproduisant à l'infini. Si le cycle correspond à une observation par fractions d'un phénomène continu en tenant compte des aléas, des dérives, des écarts... le cadre général dans lequel apparaissent ces «déviances " réorganise l'ensemble lorsque la boucle se referme sur elle-même. Dans le cycle, la course du temps est censée être immobile puisque réitérative.

3 Le cycle s'ancre dans le «fondamental », le « naturel », l'« inné », le donné une fois pour toutes. Les sens que l'on peut donner au trajet qui va de la naissance à la mort sont stabilisés et stéréotypés, réduisant la biographie à une chronologie prévue de comportements. Le cycle donné comme tracé fondamental à la mesure d'une vision, aujourd'hui dépassée, de la cosmologie, nourrit en fait des modes de représentation ambigus où les changements qui ponctuent le déroulement du cycle ne plaident en 
dernière analyse qu'en faveur d'un déterminisme originel. Chassez le naturel, il revient au galop.

\section{Les méthodes de classification et l'utilisation de la notion de cycle}

4 L'utilisation la plus dominante de l'idée de cycle est celle de l'avancée en âge. Le point de départ de tout ce qui va suivre c'est la date individuelle de naissance, non pas la date bien implantée dans l'historique ou le sociologique mais la venue au monde biologique. La répartition par âges ou catégories d'âges suppose résolu un consensus social sur la valorisation du parcours humain. Deux interprétations sont possibles : ou bien chacun des âges est une variable neutre et interchangeable, ou bien la dynamique de valorisation $\mathrm{du}$ parcours humain est linéaire et relativement stabilisée. La représentation méthodologique par âges suppose durant le temps de l'analyse l'inertie des rapports sociaux qui modifient sans cesse le contenu social des âges. Mais au nom du biologique certains auteurs ont assimilé « indicateur » et « variable »...

«Linton puis Parson ont souligné qu'un statut peut être attribué selon des critères pour ainsi dire naturels et objectifs, l'âge et le sexe appartiennent à cette catégorie ${ }^{1}$ .»

5 Tout parcours de vie témoigne d'abord des rapports sociaux dans lesquels toute démarche humaine est impliquée. La valorisation des trajectoires pour chacun des sexes n'a ni le même profil ni le même contenu et les jalonnements par années d'âge ne sont pas interchangeables.

Citons C. Baudelot :

«Le statisticien est ici trahi, à la fois par ses mots et par la représentation graphique qu'il a lui-même élaborée. En reliant par des segments de droite des points correspondants aux salaires perçus la même année par des individus d'âges différents, et en ne considérant dans le commentaire que les variations du salaire selon l'âge, on tend progressivement à oublier (et à faire oublier) qu'il s'agit, pour chaque tranche d'âge, d'individus physiquement distincts. La notion d'âge change alors de sens ; elle n'est plus entendue pour ce qu'elle est : une variable permettant de répartir une population de salariés entre plusieurs tranches d'âge. Elle tend à devenir dans l'esprit du lecteur une étape dans le cycle de vie des mêmes individus. La variable statistique "tranche d'âge" se métamorphose en âge individuel. On est ici en présence d'un bel exemple de ce que Gaston Bachelard appelait un obstacle épistémologique ${ }^{2}$."

7 Trahi par les mots et par la représentation graphique... le statisticien souligne combien une représentation graphique induit un usage social (et idéologique). Le cycle en est l'illustration la plus achevée. La représentation cyclique correspond à une vision panthéiste du monde et à un archaïsme des modes d'expression du spatio-temporel. D. Landes rappelle que les origines des mesures du temps sont liées à une cartographie céleste assez fruste, les mesures du temps ont évolué avec l'usage social qui en a été fait ${ }^{3}$. Le temps industriel taylorien et ses mesures évoluent sous nos yeux devant les avancées technologiques. L'avancée en âge ne peut se concevoir sous forme de «cycle de la vie " (de famille) que dans la mesure où les âges sont totalement abstraits des disciplines sociétales qui les construisent. La méthode n'est pas en cause, ce serait confondre l'outil et l'ouvrier; la méthode est un parti pris qui a sa propre légitimité. Si le statisticien est trahi, ce n'est ni par les mots ni par la représentation graphique en elle-même. Si l'âge 
n'est plus une fiction utile méthodologiquement, la balle est dans le camp des sociologues qui ont pris l'ombre pour la proie.

8 Réduire la complexité des mouvements qui animent le social à des modes de présentation méthodologiques (qui sont par essence des fictions réductrices) n'est pas un simple obstacle épistémologique; c'est la négation même de la démarche sociologique. Toute proposition méthodologique porte en elle un réel pouvoir d'élucidation, mais elle trace ses propres limites et ne vise pas à s'ériger en système explicatif.

\section{Le cycle, concept, idéologie}

Il est de bon ton de ne pas tirer sur une ambulance mais le cadavre quoique moribond est loin d'être enterré; son agonie est lente pour des raisons évidentes de parti pris idéologique. À partir d'une analyse des mouvements démographiques, H. Le Bras intitulait un article "Le cycle de la vie familiale, une nouveauté déjà périmée ${ }^{4}$ : il $\mathrm{y}$ remettait en cause le "calendrier» stéréotypé proposé comme structurant toute dynamique familiale. Le concept de « cycle de la vie familiale » avait déjà été dénoncé par ceux qui s'en étaient le plus servi (les sociologues de la famille), en particulier les sociologues américains, au cours d'un colloque tenu à Paris en 1973. Ce colloque se place aux prémices d'une évolution radicale de l'approche «familialiste », de la représentation parsonienne des "partages de rôles » dans la famille concourant à l'occultation des spécificités du féminin dans la sphère productive. Ce qui resurgit à travers le recours au "cycle de la vie familiale », c'est un discours idéologisé du rôle de la femme dans la société par rapport à la reproduction 5 .

Le recueil d'articles intitulé Cycles de vie et générations ${ }^{6}$ fait le point sur l'articulation entre les données transversales (à une date donnée) et l'observation longitudinale (les faits successifs pour une génération donnée). La difficulté pour le statisticien est amplifiée lorsque l'observation englobe plusieurs générations mais des propositions mathématiques et informatiques permettent d'espérer que la volonté de capter le mouvement est possible. Le projet commun de l'ouvrage était, à partir de plusieurs entrées (statistiques, économiques, psychosociologiques) de confronter la définition du Ménage (au sens statistique) au Temps (entendu dans le sens des dynamiques de la durée). Dans la préface, on annonce qu'il est question de ce qui... se déploie, se transmet, se meut, apparait, s'accumule, se prévoit, s'enrichit, succède, vieillit, dure, est en progrès, s'hérite, éclate, rétrécit... tout ce qui s'inscrit dans la durée. Mais pourquoi utiliser le mot « cycle»?

11 Dans le corps de l'ouvrage le mot «cycle» est associé au mot "vie». Ce n'est plus le «cycle» du groupe familial qui est en cause mais la durée de vie humaine. L'aspect réitératif du cycle se perd dans les sables, sauf lorsqu'il est question de générations successives. Le cycle retrouve alors son rôle pervers puisqu'il induit une succession linéaire, chaque génération faisant irruption alors que la précédente disparait. Nous sommes alors renvoyés à l'image physique «naturaliste » de la génération, bien loin des définitions sociologiques basées sur la notion de socialisation commune par groupes de pairs tels que F. Godard le propose ${ }^{7}$. Il va de soi que la "génération " de la guerre d'Algérie est une combinaison entre la classe (d'âge du service militaire obligatoire) et les hommes socialisés par une expérience forte commune. 
$12 \mathrm{Au}$ sens commun, une génération est repérée par un invariant qui est l'âge chronologique. Dans Cycles de vie et générations, toutes les difficultés méthodologiques proviennent de la trop grande rigidité de ce mode de classement, trop lourd d'images qui évoquent des étapes du parcours de vie. D’ailleurs le livre est illustré par une image d'Épinal, "Les degrés des âges ", où effectivement la durée humaine est tronçonnée en paliers qui vont de dix en dix, de la puérilité à la décrépitude. On constate alors ce que les textes dénoncent, d'une part, un jalonnement mécanique et, d'autre part, des stéréotypes de valorisation des séquences du cours de vie. Le cycle de vie est une courbe avec un apogée préconstruit et un vieillissement social parallèle au vieillissement physique. Chassez l'image qui se profile derrière les mots, elle revient au galop 8 .

\section{La valorisation des âges de la vie}

Dans mon étude en cours, la méthode biographique permet de capter des mécanismes sociaux par quoi se mettent en place de nouveaux profils des âges. Le mode d'expression dans les discours est volontiers éthique et exprime le plus souvent un certain désarroi devant l'évolution contemporaine des systèmes de dévalorisation d'âges situés antérieurement et postérieurement à un apogée adulte qui va en se rétrécissant. Il peut sembler hasardeux d'aborder une critique de la notion de «cycle » dans le parcours de la saga familiale sous l'angle de l'éthique, mais l'aspect culturel et moral des interactions de socialisation des âges dans le cadre familial permet de présenter de manière synthétique ce qui peut aussi bien se démontrer par des données chiffrées de valorisation marchande des âges salariaux...

En quoi un certain âge est-il vécu comme adéquat à l'usage qui en est fait par chacun des acteurs en présence dans le cercle familial ? Est-il temps? Est-il trop tard? Et pour qui ? Pour quoi ? Une certaine éthique du déroulement du cours de la vie est toujours présente dans les récits biographiques. Une «loi du genre» impose des opinions sur ce qui est advenu au fil du temps par rapport à un « bon usage » des âges de la vie.

La valorisation éthique des temporalités du social est fluctuante, elle est un indicateur qui témoigne des contradictions des rapports sociaux (donc économiques...) hétérogènes et parfois transitoires. Les éléments du système de valorisation des âges situe un moment de l'histoire des rapports sociaux. La synchronisation des modalités de valorisation en est toujours inachevée. Des jalonnements stéréotypés figent le mouvement sociétal qui « reconnaît» la légitimité de tel ou tel comportement au nom du critère d'âge. Face aux règles, une autonomie relative des individus impulse une dynamique d'accommodement des comportements. Des inflexions extérieures aux règles familiales infléchissent les attitudes mais le mouvement général de déstructuration du système de valorisation des âges est constamment réinterprété dans la famille.

Mon étude porte sur les récits croisés de frères et de sœurs nés dans les années 1960. L'âge des parents oscille autour de la soixantaine, l'âge est généralement moins avancé pour la mère que pour le père. Quant à leurs enfants, choisis délibérément de l'un et l'autre sexe, quelle que soit par ailleurs la composition de la fratrie, ils sont très proches dans l'âge (ce qui n'exclut pas le rapport aîné/cadet). Le double statut d'ainesse (que l'on soit fille ou garçon ne renvoie pas au même profil d'aînesse) est corrélatif du statut sexué des âges de parentalité pour les hommes et pour les femmes et plus généralement au statut sexué des âges à divers niveaux où nous les observons. De même que les âges ont 
des aspects sexués par rapport au marché du travail, dans le matrimoniat, les âges sont sexués dans la relation fraternelle : aîné/cadette n'est pas équivalent à aînée/cadet.

Dans cette étude très précisément ancrée à différents titres - contemporaine, urbaine, ciblée sur les couches moyennes, confrontant des âges entre générations à un carrefour précis de la saga familiale - l'invariant que constitue généralement le critère chronologique d'âge n'est pas opératoire en soi. Il s'agit justement de saisir, à partir des matériaux recueillis auprès de chaque individu, la variété des « marges discrètes » mises en œuvre par chacun des protagonistes d'un collectif (la famille) par rapport à une normativité globale qui peut être ce jalonnement stéréotypé qu'est l'image du « cycle». Mais le «cycle » est un outil construit par le sociologue; dans les récits individuels il s'agit de déroulement du cours de vie.

Père, mère, fils, fille : nous sommes en présence de quatre cas de figure qui vont réagir entre eux, aux fins d'une réinterprétation des règles sociales de discipline, des rythmes, des durées, des jalonnements dominants... tous les repères temporels qui construisent cette éthique du parcours de vie. L'âge d'apogée salarial du père ou son déclin professionnel de préretraité est face à l'âge de « démobilisation » maternelle de la femme ou de sa plénitude salariale... les âges de l'un et de l'autre peuvent être situés dans la même tranche d'âge, chaque « âge » est un indicateur de multiples niveaux de contraintes sociales et un même chiffre de part et d'autre recouvre deux positionnements sociaux de l'âge, de ses « droits ", de sa "valeur », de sa " dépréciation »... tous éléments socialement élaborés dans un jeu continu d'interactions. Il en va de même pour le frère et pour la sœur. Chaque parcours est dépendant des rapports sociaux qui le construisent.

En ce sens, repérer l'élaboration de l'éthique sexuée des parcours de vie qui donne lieu dans la famille à un « travail » collectif d'ajustement, très repérable dans les pratiques de la vie quotidienne, peut aider à une meilleure compréhension des mouvements de lente évolution de la valorisation sociétale des âges dans le parcours de vie. «La sociologie doit chercher ses problèmes, non dans la matière de la vie sociale, mais dans sa forme [...] un moyen de s'objectiver est de s'incorporer dans des objets impersonnels qui la symbolisent 9.» Mais l'univers symbolique ne surgit pas du néant, il se crée et se recrée et agit en «imaginaire actif». Cet imaginaire actif s'ancre et se transmet à travers des représentations et nous voilà renvoyés au pouvoir évocateur du « cycle ».

20 À un moment de la réflexion sociologique qui remet en cause un système longtemps dominant de la perception du temps, tout un pan de la réflexion sur les dynamiques de la durée se situe ailleurs que dans la valorisation taylorienne des temps sociaux ${ }^{10}$. Le " cycle de la vie familiale» stéréotypé est l'exemple même d'une tentative de nier les interactions entre la sphère de la production et celle de la reproduction. Or toute entrée dans le sujet par la famille pose de manière pressante pour le sociologue la nécessité d'analyser les effets de la simultanéité des pressions sociales qui s'exercent à l'un et l'autre titre.

21 Il reste à travers les récits croisés des membres d'un même micro-groupe social tel que le cercle familial à saisir les modalités d'une réinterprétation à la fois individuelle et collective qui s'élabore à propos des critères d'âge et l'éthique des parcours de vie. «Tout le monde sait qu'il y a une cohésion sociale dont la cause est dans une conformité des consciences particulières à un type commun qui n'est autre que le type psychique de la société11. . C'est pourquoi nous posons la question d'un hypothétique consensus familial sur la socialisation des parcours de vie. Notre étude nous montre, à travers les récits autobiographiques, que la démarche est collective et constamment recommencée. Elle 
mobilise chacun dans le but commun de conforter à la fois des parcours individuels et une saga familiale. Toute famille brode sur un canevas qui s'élabore ailleurs mais traverse la famille. L'apport spécifique des récits de vie est de capter tous les mouvements de réorganisation, de détournement et de réinterprétation.

\section{NOTES}

1. Cité dans R. Boudon \& F. Bourricaud, Dictionnaire critique de la sociologie, Paris, Presses universitaires de France, 1982.

2. C. Baudelot, "Effets d'âge et de génération dans l'évolution du salaire individuel », in D. Kessler \& A. Masson, eds, Cycles de vie et générations, Paris, Économica, 1985, p. 9-24.

3. D. S. Landes, L'heure qu'il est. Les horloges, la mesure du temps et la formation du monde moderne, Paris, Gallimard, 1987 («Bibliothèque illustrée des histoires »).

4. H. Le Bras, «Le cycle de la vie familiale, une nouveauté déjà périmée ", in Les cycles de la vie familiale, $\mathrm{n}^{\circ} \mathrm{sp}$. de : Dialogue, 71, 1981.

5. Cf. les travaux de l'Atelier production reproduction (Paris), Séminaire PIRTTEM, 1986-1988.

6. D. Kessler \& A. Masson, eds, Cycles de vie et générations.

7. F. Godard, «Cultures et modes de vie : de génération en génération », in F. Proust, ed., Les jeunes et les autres. Contributions des sciences de l'homme à la question des jeunes, Vaucresson, Centre de recherche interdisciplinaire de Vaucresson, 1986.

8. Cf. Annette Langevin, " Pour une nouvelle définition des âges de la vie », in C. Valabrègue, C. Berger-Forestier \& A. L., Ces maternités que l'on dit tardives, Paris, Laffont, 1982, p. 125-217 («Réponses »). J'ai relevé qu'il n'existe pas une seule image d'Épinal sur les « degrés des âges », mais trois dont la confrontation est riche d'enseignements pour le sociologue qui s'intéresse au cognitif.

9. G. Simmel, « Comment les formes sociales se maintiennent ", L'Année sociologique, 1, 1896-1897, p. 71-79.

10. A. Langevin, "La synchronisation des temps sociaux : des dynamiques et des familles ", in $L a$ dynamique familiale et les constructions sociales du temps, Actes du Colloque (Université de Liège, 7-8 mai 1987); Eviatar Zerubavel, Hidden rhythms. Schedules and calendars in social life, Chicago, University of Chicago Press, 1981.

11. E. Durkheim, La division du travail social, Paris, PUF, 1967. 